\title{
The effect of different running shoes on treadmill running mechanics and muscle activity assessed using statistical parametric mapping (SPM)
}

\author{
Corina Nüesch, Ph.D. ${ }^{1,2}$ \\ Elena Roos, M.Sc. ${ }^{1}$ \\ Christian Egloff, M.D. ${ }^{1}$ \\ Geert Pagenstert, M.D. ${ }^{1}$ \\ Annegret Mündermann, Ph.D. ${ }^{1,2}$ \\ ${ }^{1}$ Department of Orthopaedics and Traumatology, University Hospital Basel, Spitalstrasse 21, \\ 4031 Basel, Switzerland \\ ${ }^{2}$ Department of Biomedical Engineering, University of Basel, Gewerbestrasse 14, 4123 Basel, \\ Switzerland \\ ${ }^{2}$ Department of Clinical Research, University of Basel, Schanzenstrasse 55, 4031 Basel, \\ Switzerland
}

Original Article

December 2018

Gait Posture. 2019 Mar;69:1-7. doi: 10.1016/j.gaitpost.2019.01.013. Epub 2019 Jan 10.

Address of correspondence: Prof. Dr. Annegret Mündermann, Ph.D.

Research Director Functional Biomechanics

Department of Orthopaedics and Traumatology

University Hospital Basel

Spitalstrasse 21

4031 Basel, Switzerland

Tel. +41613285445

Email annegret.muendermann@unibas.ch

Acknowledgements: Shoes for this study were provided by On AG. The sponsor was not involved in the study design, data analysis or interpretation.

Word count: 2551 words

Abstract word count: 294 words

Short title: Running shoe biomechanics and SPM 


\begin{abstract}
Background. Difference in joint mechanics between running shoes are commonly assessed using discrete parameters, yet statistically significant differences in these parameters between shoes are often scarce with small effect sizes. Statistical parametric mapping (SPM) has been suggested as suitable method for analyzing one-dimensional data such as kinematic, kinetic or muscle intensity time series.
\end{abstract}

Research question. The purpose of this study was to determine differences in treadmill running mechanics between novel running shoes using SPM.

Methods. Joint kinematics, muscle activity and ground reaction force were assessed in 19 rearfoot runners in their own shoes and in two test shoes during treadmill running (test shoe 1: 13 distinct rubber elements in the outer sole, springboard within EVA midsole with posterior elements shifted anteriorly by approximately $1.5 \mathrm{~cm}$; test shoe 2: 17 distinct EVA elements with conventional heel geometry). Joint kinematics were measured using an inertial sensor system, and ground reaction force was measured using an instrumented treadmill. Results. SPM analysis with repeated measures ANOVA revealed significant reductions in the ankle angle and in tibialis anterior, peroneus longus, vastus medialis and lateralis muscle activity during weight acceptance and in peroneus longus muscle activity during early and late swing and in semitendinosus muscle activity during late swing for the test shoes. Significant differences in muscle activity were observed in the interval of the main activity of the respective muscle. SPM on individual data revealed statistically significant and relevant within-subject differences between conditions in kinematic, muscle activity and ground reaction force patterns.

Significance. Inertial sensor systems and SPM may provide an efficient way of detecting changes in joint mechanics between running shoes within runners. Detecting within-subject 
differences in running mechanics between conditions not only requires statistical criteria but also criteria on the relevance of the magnitude of differences.

Keywords: running shoes; statistical parametric mapping; electromyography; joint kinematics; ground reaction force 


\section{Introduction}

Running shoes are characterized by the geometric and material properties of the sole construction and the shoe upper that are known to affect running biomechanics. ${ }^{1,2}$ Recently, a shoe design has been introduced to the market comprising open interlocking rubber components on the outer sole in the heel and forefoot regions presumably cushioning landing and providing firm push-off. An early model of this novel shoe had little effects on selected ground reaction force parameters but shifted the center of pressure compared to runners' own conventional running shoes. ${ }^{3}$ Other modifications in sole design have resulted in changes in foot and leg kinematics ${ }^{4}$, kinetics ${ }^{2,5,6}$ and in inconsistent shoe-induced changes in muscle activity between runners. ${ }^{7-9}$ Similarly, we expect that the novel shoe design will also cause changes in running biomechanics.

Assessing joint kinematics and kinetics and lower extremity muscle activity during running has been important not only in the development of novel footwear ${ }^{2,10-12}$ but also in understanding the development and risk of injuries. ${ }^{13-15}$ These time series are characterized by their cyclic patterns and often described by few discrete parameters, i.e. zero-dimensional metrics, such as peak values or range of motion ${ }^{1,13-15}$ assuming that these parameters are functionally relevant. However, discrete parameters do not capture important aspects of time series such as the shape of the pattern and hence are limited in their ability to detect differences between conditions or populations. Other approaches considering the entire times series apply complex statistical methods such as principal component analysis. ${ }^{16-18}$ Results of such analyses are difficult to interpret because the principle axes do not coincide with anatomical or functional axes. Recently, statistical parametric mapping (SPM) has been suggested as suitable method for analyzing one-dimensional (1D) data such as kinematic, kinetic or muscle intensity time series and shown to be superior to the over-simplification of 
discrete parameter analyses. ${ }^{19}$ For instance, SPM has been used to detect differences in knee kinematics between patients after anterior cruciate ligament injury and healthy control subjects $^{20}$, gait compensations after tibiotalar arthrodesis ${ }^{21}$, differences in muscle intensity during walking between age groups ${ }^{22}$ and differences in ground reaction force between running at different running speeds. ${ }^{19}$

In recent years, inertial measurement units (IMUs) have gained popularity for assessing joint kinematics during running. Advantages of such systems include their ease of use, high efficiency and ability to measure data in free-living as opposed to laboratory environments. Kinematic waveform patterns measured with these systems are comparable to those measured using standard instrumented 3-dimensional gait analysis with reflective markers and highspeed cameras. ${ }^{23,24}$ Moreover, spatiotemporal and discrete kinematic parameters computed by IMU systems have shown good within and between day reliability. ${ }^{23,25}$

The purpose of this study was to determine differences in treadmill running mechanics between novel running shoes using SPM. We hypothesized that waveforms of joint kinematics, ground action force and electromyographic (EMG) signals differ between the tested running shoes.

\section{Materials and methods}

Nineteen habitual rearfoot runners with a minimum weekly running distance of $20 \mathrm{~km}$ to prevent possible fatigue during the test (11 female; age, $27.7 \pm 8.6$ years; height, $1.75 \pm 0.08$ m; body mass, $66.1 \pm 12.3 \mathrm{~kg}$; body mass index, $21.6 \pm 2.5 \mathrm{~kg} / \mathrm{m}^{2}$; weekly running distance, $42.3 \pm 18.6 \mathrm{~km}$ ) participated in this study after providing informed consent. This study was 
approved by the institutional review board and conducted in accordance with the Declaration of Helsinki.

Written informed consent was obtained prior to participation. Participants were able to familiarize with treadmill running at their self-selected running speed that was kept constant for all shoe conditions. Surface electrodes were placed bilaterally. Inertial sensors were attached to the lower extremity and pelvis. Subsequently, participants walked for 1 minute and ran for 5 minutes with the three different shoes while kinematic, EMG and force data were collected. The study was designed as a modified block design. Participants first wore their personal running shoes to ensure capturing their habitual running mechanics (mean mass: $274 \pm 42 \mathrm{~g}$; mean drop: $10.1 \pm 2.2 \mathrm{~mm} ; 13$ training and 6 competition; 6 stability and 13 neutral; 13 posterior heel flare). Subsequently, two test shoes (both On AG, Zürich, Switzerland; Figure 1) were tested in randomized order. The Cloudsurfer (260 g, drop: 7.0 mm; training; neutral) comprises 13 distinct rubber elements in the outer sole and a springboard within the EVA midsole where the posterior elements are shifted anteriorly by approximately $1.5 \mathrm{~cm}$ compared to a standard running shoe. The Cloud (165 g, drop: $6.0 \mathrm{~mm}$; training; neutral) comprises 17 distinct EVA elements with heel geometry similar to that of conventional running shoes.

Sagittal plane joint angles of the ankle, knee and hip were measured using the RehaGait ${ }^{\circledR}$ system (Version 2.0.7.2; Hasomed GmbH, Magdeburg, Germany) consisting of seven mobile inertial sensors and analysis software. ${ }^{23}$ Sensors were firmly attached bilaterally to the lateral aspect of the heel, shank and thigh using Velcro belts. One sensor was attached to the skin overlying L4 using double-sided tape to ensure secure location on the skin. The system was calibrated during a 10-s standing trial and a 10-s slight squatting movement. Kinematic 
patterns for running including information on the time of heel-strike were exported for further analysis. Kinematic data were cut at heel-strike, and all steps time normalized to the gait cycle (101 data points per gait cycle).

Simultaneously with the kinematic measurements, EMG data were collected using a 14channel EMG system (Biovision, Wehrheim, Germany; sampling rate, $2400 \mathrm{~Hz}$; bandwidth, 10-700 Hz; gain range, 1000-5000). Bipolar round $\mathrm{Ag} / \mathrm{AgCl}$ surface electrodes (Noraxon U.S.A. Inc., Scottsdale, AZ, USA; 10-mm diameter, 20-mm inter-electrode distance) were placed bilaterally on the tibialis anterior, gastrocnemius medialis and lateralis, peroneus longus, vastus medialis and lateralis, and semitendinosus muscles according to the European recommendations for surface EMG (SENIAM) ${ }^{26}$ after shaving and cleaning the skin with alcohol. To reduce movement artifacts, amplifiers and cables were taped to the skin. The ground electrode was placed on the left tibial tubercle. EMG signals were recorded for the treadmill running trials and high-pass filtered $\left(4^{\text {th }}\right.$ order Butterworth filter; cut-off frequency, $20 \mathrm{~Hz}$ ). After full-wave rectification, the moving average was calculated with a time constant of $41.7 \mathrm{~ms}$. EMG intensities for each muscle were normalized to the average of the maximum EMG activity of all trials during running with the participant's personal shoes. EMG intensity traces were cut at heel-strike, and all steps time normalized to the gait cycle (101 data points per gait cycle).

Ground reaction force between the foot and the treadmill were captured using an instrumented treadmill with an embedded plantar pressure plate $(\mathrm{h} / \mathrm{p} / \mathrm{cosmos}$, Zebris FDM-T; 7168 sensors; area, $1.5 * 0.5 \mathrm{~m}^{2}$; range, $1-120 \mathrm{~N} / \mathrm{cm}^{2}$; precision, $1-120 \mathrm{~N} / \mathrm{cm}^{2} \pm 5 \%$; sampling rate, $120 \mathrm{~Hz}$ ). The shoe print was divided into three regions: heel, midfoot and forefoot. The vertical ground reaction force was calculated for the entire contact area and 
normalized to body weight. Force data for each step were time normalized to the gait cycle (101 data points per gait cycle).

We tested the null hypothesis that there are no differences in 1D biomechanical data between the different running shoes by statistically examining the entire times series using SPM. All SPM analyses were conducted in Matlab (MathWorks Inc., Natick, MA, USA) using the open-source software package spm1D $0.4^{27}$ (www.spm1d.org). Between-condition statistical analyses were conducted as described by Pataky. ${ }^{27}$ Briefly, repeated measures analyses of variance (ANOVA) were performed on all three conditions with post hoc paired t-tests to compare condition pairs. The null hypothesis was rejected if the experimentally computed tvalue for trajectory 1D data exceeded the critical value that smooth, 1D multivariate Gaussian data would reach in an infinite number of experiments involving smooth 1D data. For each subject and condition, data for 20 steps were available and the mean trajectory was computed. We detected differences between shoes for the entire group. In addition, we detected differences between conditions for each subject and 1D parameter. The significance level for all statistical tests was set a priori to 0.05 . A relevance criteria was defined as the root mean square error of repeated measurements for the same condition. ${ }^{23}$ Statistically significant differences in 1D force and EMG patterns were considered relevant if the difference between conditions were greater than $5 \%$.

\section{Results}

Cadence and stride duration did not differ significantly between shoes (Table 1). Running speed was kept constant for all test conditions and was $2.98 \pm 0.39 \mathrm{~m} / \mathrm{s}$. 
The SPM analysis with repeated measures ANOVA revealed significant differences between shoe conditions in the ankle angle and in tibialis anterior, peroneus longus, vastus medialis and vastus lateralis muscle activity during weight acceptance (Figure 2). Moreover, differences between shoe conditions were observed in peroneus longus muscle activity during early and late swing and in semitendinosus muscle and tibialis anterior activity during late swing (Figure 2).

The Cloudsurfer (interlocking elements, altered heel geometry) reduced the ground reaction force during push-off and the foot to ground angle during early swing compared to the own shoes (Figure 3). Moreover, the Cloudsurfer reduced tibialis anterior and semitendinosus muscle activity during late swing (Figure 3).

The Cloud (interlocking elements, normal heel geometry) did not change kinematic or kinetic parameters compared to the own shoes (Figure 4). However, the Cloud reduced tibialis anterior and vastus medialis and lateralis muscle activity during weight acceptance, peroneus longus muscle activity during early swing and semitendinosus muscle activity during late swing (Figure 4).

SPM on individual data revealed statistically significant differences between conditions for some participants and most parameters. Applying the relevance criteria ${ }^{23}$ substantially reduced the participant-parameter-time interval combinations with statistically significant and relevant differences between conditions. Specifically, participants showed differences between conditions based on SPM and the relevance criteria only for some parameters, and these differences did not occur in the same parameters or during the same gait phase among 
these participants (Figures 5 and 6). Systematic differences depending on the properties of the control shoes were not observed.

\section{Discussion}

The purpose of this study was to determine differences in treadmill running mechanics between novel running shoes using SPM. We hypothesized that waveforms of joint kinematics, ground reaction force and EMG signals differ between the tested running shoes. SPM on group data detected only small differences in kinematic parameters between all shoes. Interestingly, SPM revealed difference in muscle activity for several leg muscles during different phases of running. Within individuals, SPM revealed statistically significant differences between conditions for most time series during most of the gait cycle. However, the magnitude of these differences did not always achieve relevance according to predefined criteria. Overall, SPM revealed differences in running biomechanics between the running shoes tested in this study.

In recent years, SPM has been increasingly used to investigate differences in ambulatory mechanics. For instance, Tam et al. ${ }^{28}$ observed differences in sagittal plane kinematic at the knee during late stance and swing and at the ankle during early stance, mid-stance and swing phase, differences in vertical ground reaction force during early stance but no differences in EMG signals of selected muscles between barefoot and shod running in habitually shod runners. Similarly, SPM did not reveal posthoc differences in EMG intensity of selected lower leg muscles during walking between young and adult groups in a study by Robinson et al. ${ }^{22}$. These results are in contrast to differences in muscle activity between running shoes observed in our study. Sole et al. ${ }^{20}$ applied SPM to detect differences in knee trajectories during stair descent between participants with previous anterior cruciate ligament rupture 
who underwent reconstruction or only physical therapy and healthy controls. Participants who received only physical therapy had a combination of less flexion at initial foot contact and less adduction during weight acceptance than healthy controls, but stair walking mechanics did not differ between patients after reconstruction and healthy controls. Reported differences in these studies were mostly below $3^{\circ}$, and none of these studies applied clinically or functionally based criteria to determine the relevance of observed differences.

SPM detected some kinematic differences between shoe conditions. However, after applying the additional relevance criteria, these condition differences vanished. Interestingly, both shoes did not elicit any major changes in ankle, knee or hip kinematics although muscle activity in both shank and thigh muscles were affected. Reductions in muscle activity occurred in the interval of the main activity of these muscles, and hence are highly functionally relevant. These reductions were observed despite of constant running speed, cadence and stride time with both test shoes implying that running with these shoes should be more energy efficient ${ }^{29}$. This result is particularly interesting because few studies have studied the effects of footwear on muscle activity and most reported inconsistent shoeinduced changes in muscle activity between runners. ${ }^{7-9}$ The observed reductions in muscle activity may explain the feedback by some runners who had improved their performance or reported being able to return to running in these shoes after experiencing pain in conventional running shoes. These observations are further supported by differences in running economy when running with different running shoes as observed by Lussiana et al. ${ }^{2}$ The tibialis anterior muscle slows the foot roll-over motion during landing through eccentric contraction. Changes in the demands for eccentric contractions of the tibialis anterior may be highly relevant for some athletes. For instance, reducing eccentric activity of the tibialis anterior during landing may be beneficial in patients with chronic exertional compartment syndrome 
without having to change their strike pattern. ${ }^{30}$ Moreover, hamstring activity before landing is necessary to decelerate and control the swing of the leg (semitendinosus) in preparation for landing. Finally, peroneus activity during weight acceptance is responsible for stabilization of the ankle joint in pronation, and reductions in its activity suggest a more natural position of the ankle joint complex during this phase requiring less muscle activity.

The lack of group differences between conditions for 1D kinematic data may be explained by individual differences in these data between conditions. In fact, individual data showed very consistent differences in kinematic, EMG and force patterns within subject and condition. Moreover, the experimental conditions elicited different kinematic responses in different runners with consistent changes in muscle intensity across subjects. These results partially confirm previous data of subject specific responses to running footwear. ${ }^{31}$ Within-subject SPM analysis in our study clearly showed that running shoes affect biomechanical patterns during the entire gait cycle and not just specific aspects of these patterns. Hence, this method may be appropriate for determining kinematic responders and non-responders to specific shoe conditions.

The data presented in this study are novel because we applied SPM to detect differences in kinematic, kinetic and EMG parameters between running shoes. Multiple tests were conducted but we did not adopt a correction for multiple comparisons. This was done because this study did not aim to test specific predictions regarding footwear-induced biomechanical changes. Instead this was an exploratory study aimed at detecting possible footwear-induced effects with maximum sensitivity. We acknowledge that some of these results may be false positives, and that further, predictive studies are needed to more clearly elucidate footwearrelated effects. In our study, participants wore their personal shoes that varied in construction 
and stability parameters. While differences in running mechanics between the test running shoes and the same control shoe for all subjects may have led to different results, subjects may not adopt their habitual running style in these shoes. Moreover, we only assessed sagittal plane joint kinematics using the inertial sensor system. It is possible that the test running shoes included in this study may have also affected frontal plane kinematics, which were not assessed in our study. Nonetheless, this study showed that SPM can reveal differences in muscle activity of lower and upper leg muscles between running shoe on a group and individual level. Such analysis may be useful for the evaluation and development of novel running footwear.

\section{Conclusions}

To the best of our knowledge, this is the first study to use an inertial sensor system for detecting differences in running mechanics between running shoes. Moreover, we applied SPM to kinematic data obtained using an inertial sensor system. Greater individual than group differences in running mechanics between shoes represents further evidence for individual adaptations to running footwear. SPM may be particularly useful for studying within subject changes in kinematic, EMG and ground reaction force during running. However, because of the small step-to-step variability in biomechanical parameters within subjects, detecting within-subject differences in running mechanics between conditions not only requires statistical criteria but also criteria regarding the relevance of the magnitude of differences.

\section{Conflict of interest statement}

The authors declare no conflict of interest. 


\section{References}

1. Mündermann A, Nigg BM, Humble RN, Stefanyshyn DJ. Foot orthotics affect lower extremity kinematics and kinetics during running. Clin Biomech 2003; 18(3): 254-262.

2. Lussiana T, Fabre N, Hebert-Losier K, Mourot L. Effect of slope and footwear on running economy and kinematics. Scand J Med Sci Sports 2013; 23(4): e246-253.

3. Knoepfli-Lenzin C, Waech JC, Gulay T, Schellenberg F, Lorenzetti S. The influence of a new sole geometry while running. 2014; 32(18): 1671-1679.

4. Stacoff A, Reinschmidt C, Nigg BM, Van Den Bogert AJ, Lundberg A, Denoth J, Stussi E. Effects of shoe sole construction on skeletal motion during running. 2001; 33(2): 311-319.

5. Baltich J, Maurer C, Nigg BM. Increased vertical impact forces and altered running mechanics with softer midsole shoes. 2015; 10(4): e0125196.

6. Dinato RC, Ribeiro AP, Butugan MK, Pereira IL, Onodera AN, Sacco IC. Biomechanical variables and perception of comfort in running shoes with different cushioning technologies. 2015; 18(1): 93-97.

7. Nigg BM, Stefanyshyn D, Cole G, Stergiou P, Miller J. The effect of material characteristics of shoe soles on muscle activation and energy aspects during running. $\mathbf{J}$ Biomech 2003; 36(4): 569-575.

8. Murley GS, Landorf KB, Menz HB, Bird AR. Effect of foot posture, foot orthoses and footwear on lower limb muscle activity during walking and running: a systematic review. Gait Posture 2009; 29(2): 172-187.

9. Wakeling JM, Pascual SA, Nigg BM. Altering muscle activity in the lower extremities by running with different shoes. Med Sci Sports Exerc 2002; 34(9): 1529-1532. 
10. Nigg BM, Vienneau J, Smith AC, Trudeau MB, Mohr M, Nigg SR. The Preferred Movement Path Paradigm: Influence of Running Shoes on Joint Movement. Med Sci Sports Exerc 2017;

11. Willwacher S, Potthast W, Konrad M, Brüggemann GP. Effect of heel construction on muscular control potential of the ankle joint in running. J Appl Biomech 2013; 29(6): $740-748$.

12. Cheung RT, Wong MY, Ng GY. Effects of motion control footwear on running: a systematic review. J Sports Sci 2011; 29(12): 1311-1319.

13. Nigg BM, Baltich J, Hoerzer S, Enders H. Running shoes and running injuries: mythbusting and a proposal for two new paradigms: 'preferred movement path' and 'comfort filter'. Br J Sports Med 2015; 49(20): 1290-1294.

14. Hreljac A, Marshall RN, Hume PA. Evaluation of lower extremity overuse injury potential in runners. Med Sci Sports Exerc 2000; 32(9): 1635-1641.

15. Willems T, Witvrouw E, Delbaere K, De Cock A, De Clercq D. Relationship between gait biomechanics and inversion sprains: a prospective study of risk factors. Gait Posture 2005; 21(4): 379-387.

16. Nigg BM, Baltich J, Maurer C, Federolf P. Shoe midsole hardness, sex and age effects on lower extremity kinematics during running. J Biomech 2012; 45(9): 1692-1697.

17. Foch E, Milner CE. The influence of iliotibial band syndrome history on running biomechanics examined via principal components analysis. J Biomech 2014; 47(1): $81-86$

18. Trudeau MB, von Tscharner V, Vienneau J, Hoerzer S, Nigg BM. Assessing Footwear Effects from Principal Features of Plantar Loading during Running. Med Sci Sports Exerc 2015; 47(9): 1988-1996. 
19. Pataky TC, Robinson MA, Vanrenterghem J. Vector field statistical analysis of kinematic and force trajectories. 2013; 46(14): 2394-2401.

20. Sole G, Pataky T, Tengman E, Hager C. Analysis of three-dimensional knee kinematics during stair descent two decades post-ACL rupture - Data revisited using statistical parametric mapping. J Electromyogr Kinesiol 2017; 3244-50.

21. Bruening DA, Cooney TE, Ray MS, Daut GA, Cooney KM, Galey SM. Multisegment Foot Kinematic and Kinetic Compensations in Level and Uphill Walking Following Tibiotalar Arthrodesis. Foot Ankle Int 2016; 37(10): 1119-1129.

22. Robinson MA, Vanrenterghem J, Pataky TC. Statistical Parametric Mapping (SPM) for alpha-based statistical analyses of multi-muscle EMG time-series. J Electromyogr Kinesiol 2015; 25(1): 14-19.

23. Nüesch C, Roos E, Pagenstert G, Mündermann A. Measuring joint kinematics of treadmill walking and running: Comparison between an inertial sensor based system and a camera-based system. J Biomech 2017; 5732-38.

24. Bailey GP, Harle R. Assessment of Foot Kinematics During Steady State Running Using a Foot-mounted IMU. Procedia Engineering 2014; 72(Supplement C): 32-37.

25. Donath L, Faude O, Lichtenstein E, Nüesch C, Mündermann A. Validity and reliability of a portable gait analysis system for measuring spatiotemporal gait characteristics: comparison to an instrumented treadmill. J Neuroeng Rehabil 2016; 13(1): 6.

26. Hermens HJ, Freriks B, Disselhorst-Klug C, Rau G. Development of recommendations for SEMG sensors and sensor placement procedures. J Electromyogr Kinesiol 2000; 10(5): 361-374.

27. Pataky TC. One-dimensional statistical parametric mapping in Python. Comput Methods Biomech Biomed Engin 2012; 15(3): 295-301. 
28. Tam N, Prins D, Divekar NV, Lamberts RP. Biomechanical analysis of gait waveform data: exploring differences between shod and barefoot running in habitually shod runners. Gait Posture 2017; 58274-279.

29. Abe D, Muraki S, Yanagawa K, Fukuoka Y, Niihata S. Changes in EMG characteristics and metabolic energy cost during 90-min prolonged running. Gait Posture 2007; 26(4): 607-610.

30. Diebal AR, Gregory R, Alitz C, Gerber JP. Forefoot running improves pain and disability associated with chronic exertional compartment syndrome. Am J Sports Med 2012; 40(5): 1060-1067.

31. Nigg BM, Stergiou P, Cole G, Stefanyshyn D, Mündermann A, Humble N. Effect of shoe inserts on kinematics, center of pressure, and leg joint moments during running. Med Sci Sports Exerc 2003; 35(2): 314-319. 


\section{Table}

Table 1 - Mean (1 standard deviation) of spatiotemporal parameters for the three test shoes (own shoe, Cloudsurfer, Cloud). P-values for the analysis of variance are listed.

\begin{tabular}{llllc}
\hline Parameter & Own shoe & Cloudsurfer & Cloud & P-value \\
\hline Cadence (steps/min) & $114.4(7.1)$ & $115.8(7.4)$ & $115.3(7.4)$ & .089 \\
Stride duration (s) & 1.053 & 1.040 & 1.045 & .090 \\
& $(0.065)$ & $(0.064)$ & $(0.064)$ & \\
\hline
\end{tabular}




\section{Figure captions}

Figure 1 - Photograph of the two test shoes. Top - Cloudsurfer; bottom - Cloud.

Figure 2 - Mean (1 standard deviation) patterns for kinematic, EMG and ground reaction force data for the Cloudsurfer (cyan), Cloud (red) and own shoe (black) and time-dependent F-values of the SPM (main statistical test; analysis of variance) for all subjects (dashed red lines $-\mathrm{P}=0.05$ level). Grey bars indicate regions with statistically significant differences.

Figure 3 - Mean (1 standard deviation) patterns for kinematic, EMG and ground reaction force data for the Cloudsurfer (cyan) and own shoe (black) and time-dependent t-values of the SPM for all subjects (post hoc results; dashed red lines $-\mathrm{P}=0.05$ level). Grey bars indicate regions with statistically significant differences with magnitude above the relevance criterion. $^{23}$

Figure 4 - Mean (1 standard deviation) patterns for kinematic, EMG and ground reaction force data for the Cloud (red) and own shoe (black) and time-dependent t-values of the SPM (post hoc results; dashed red lines $-\mathrm{P}=0.05$ level). Grey bars indicate regions with statistically significant differences with magnitude above the relevance criterion. ${ }^{23}$

Figure 5 - Mean (1 standard deviation) patterns for foot to ground angle for the Cloudsurfer (cyan) and own shoe (black) and time-dependent t-values of the SPM for each subject (post hoc results; dashed red lines $-\mathrm{P}=0.05$ level). Grey bars indicate regions with statistically significant differences with magnitude above the relevance criterion. ${ }^{23}$ 
Figure 6 - Mean (1 standard deviation) patterns for kinematic, EMG and ground reaction force data for the Cloudsurfer (cyan) and own shoe (black) and time-dependent t-values of the SPM for subject 15 . Grey bars indicate regions with statistically significant differences with magnitude above the relevance criterion. ${ }^{23}$ 

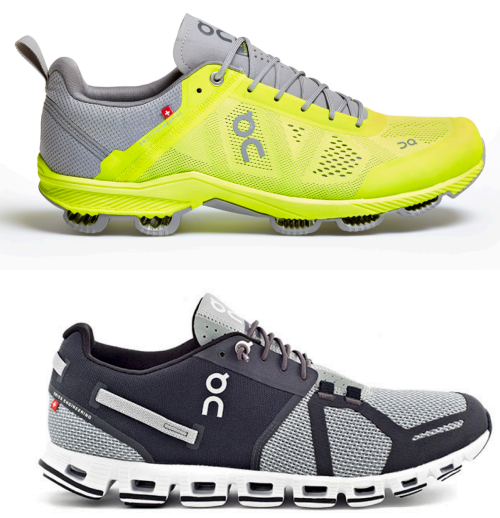

21 

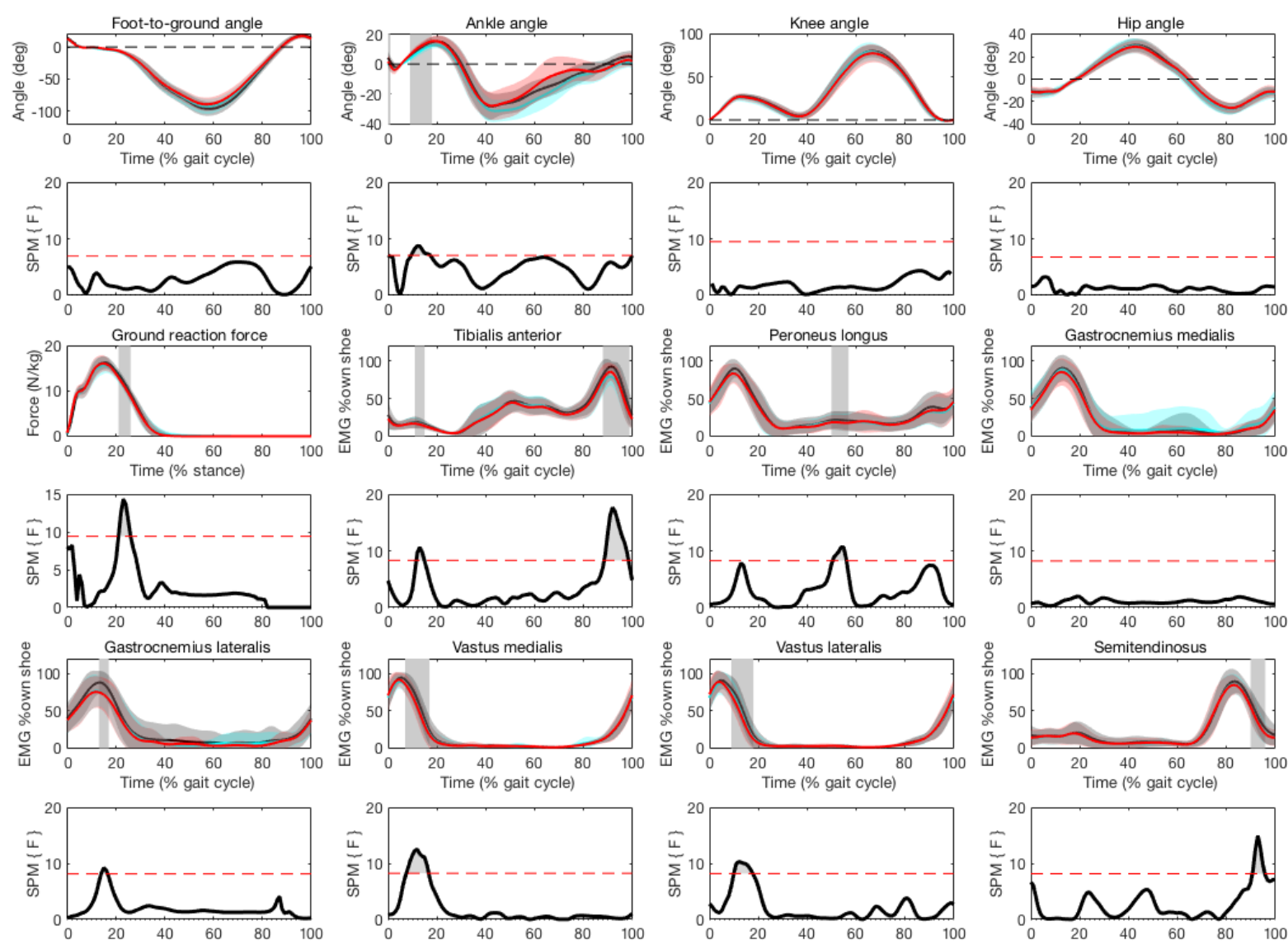

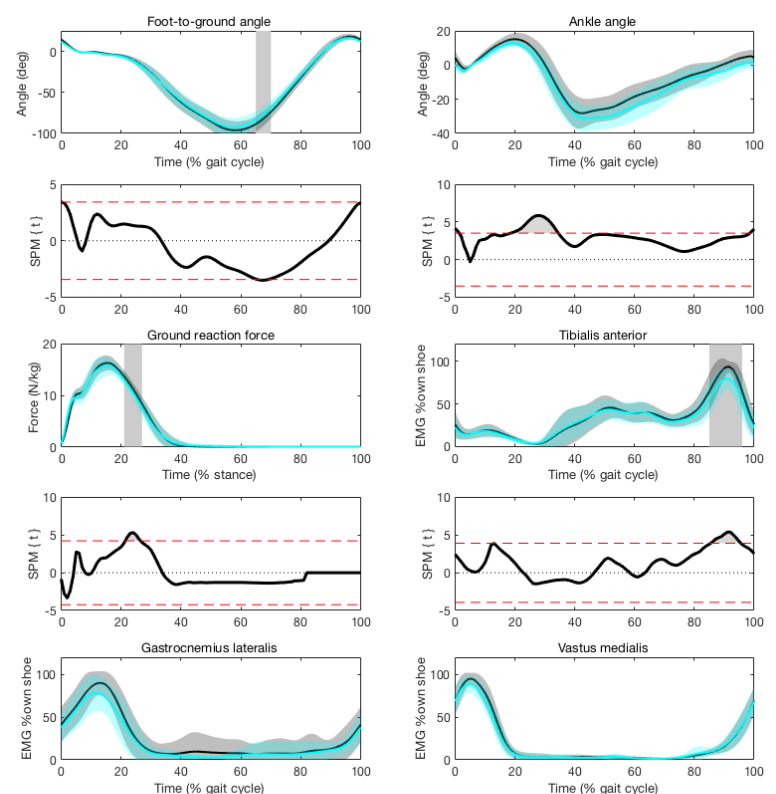

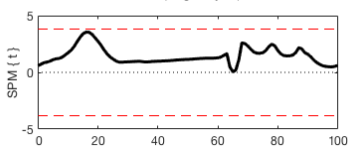

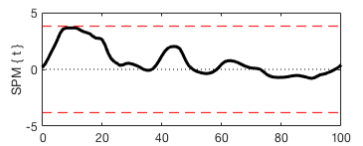

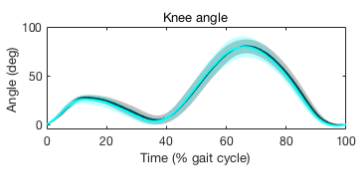
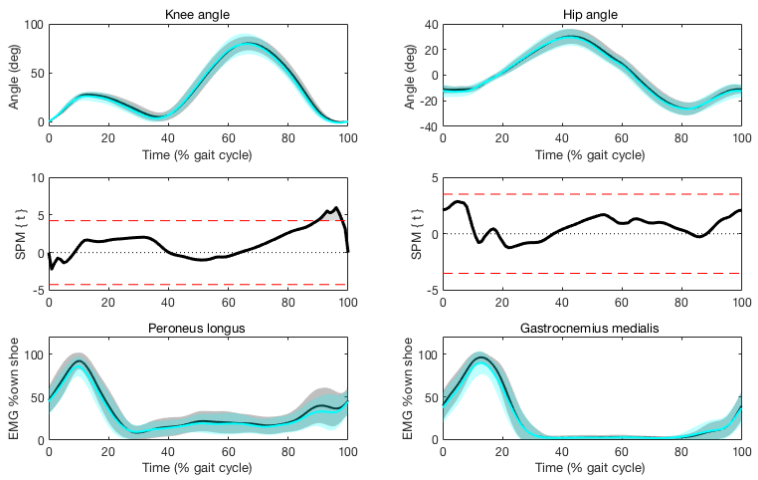
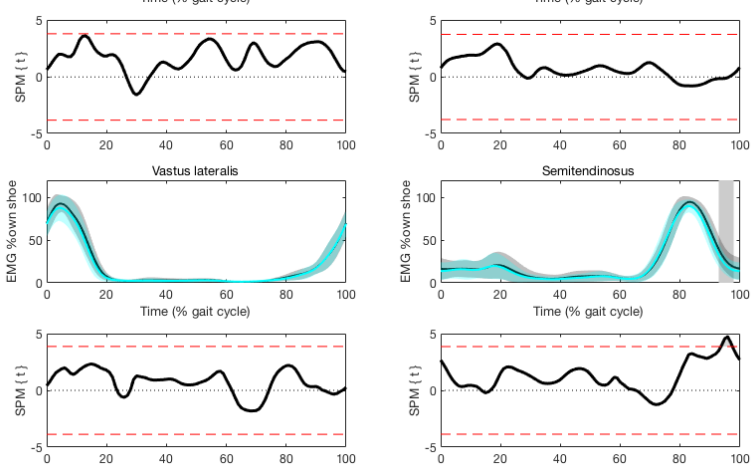

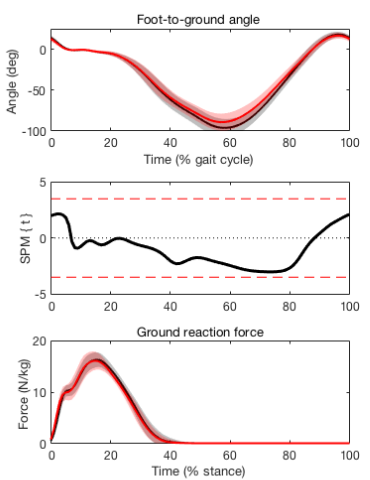

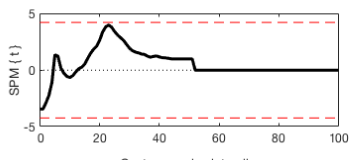
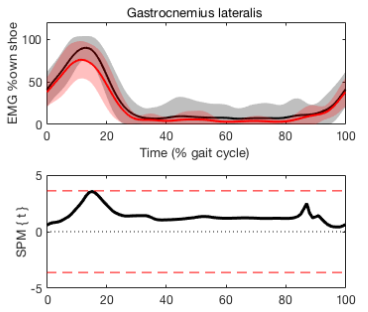
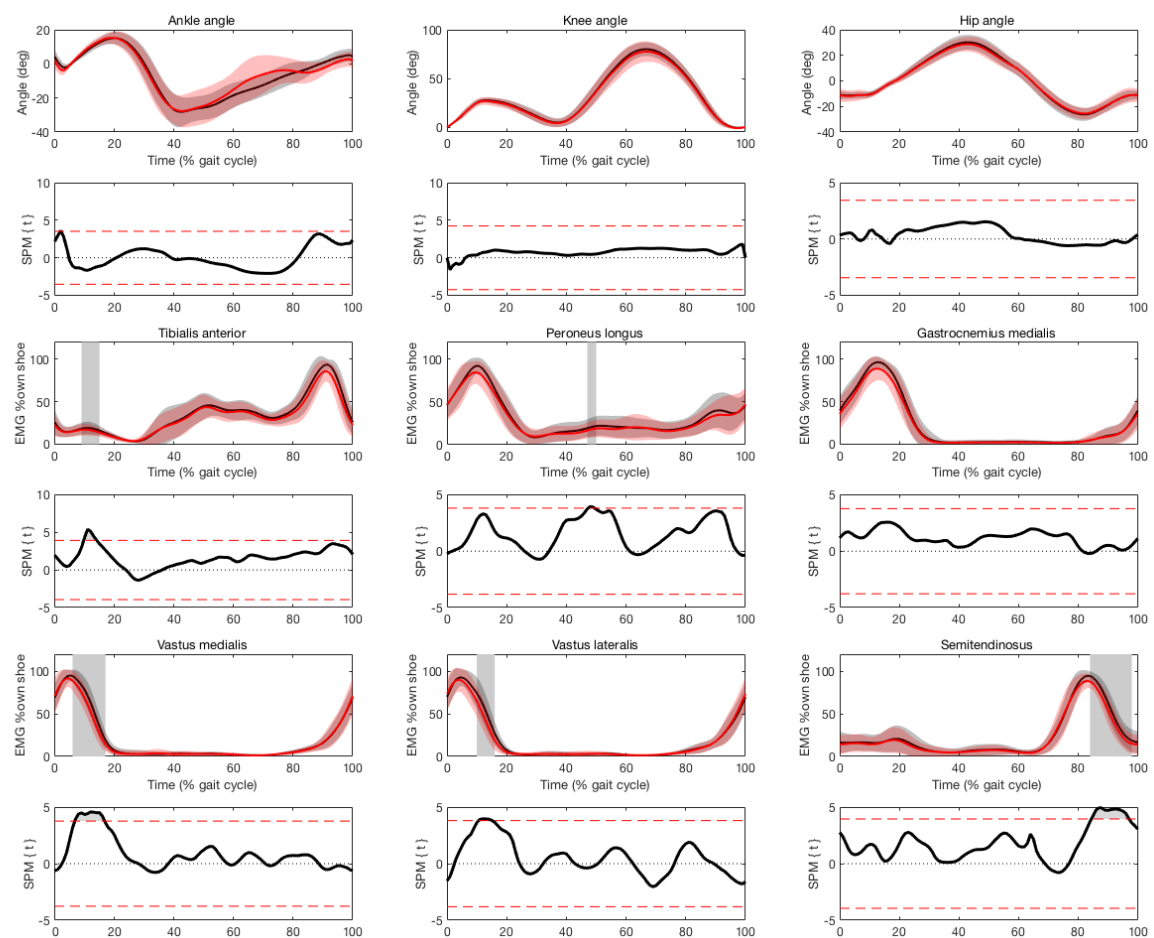

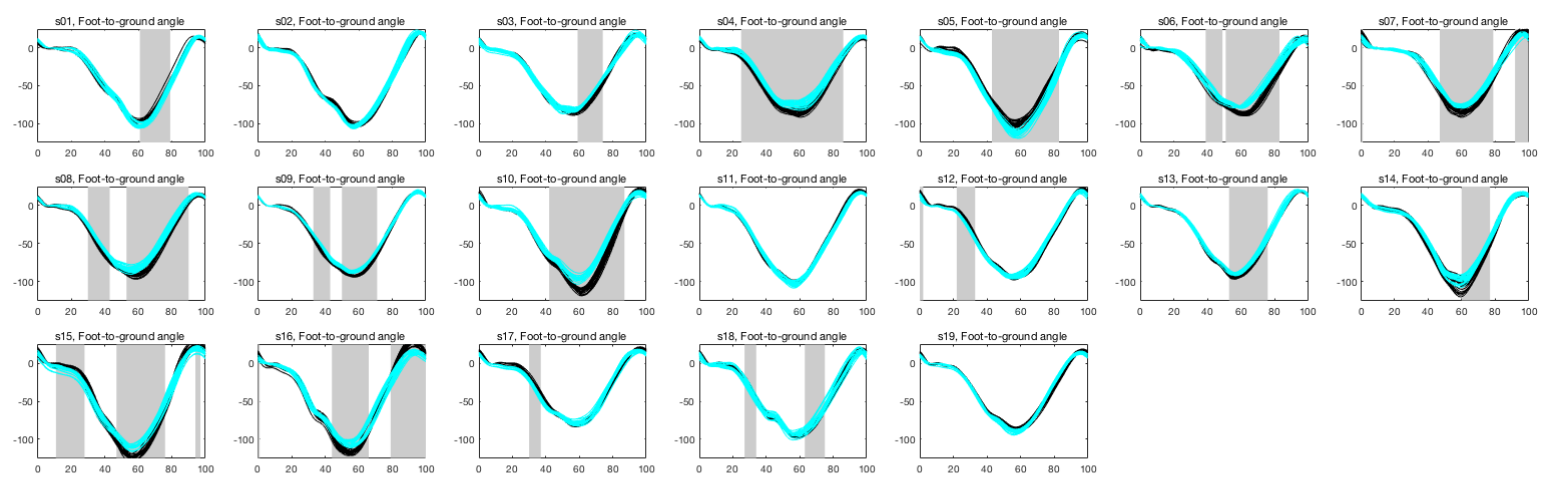

我

$\underbrace{\infty}_{-\infty}$

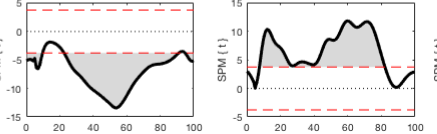

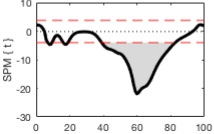

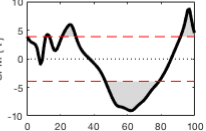

WW W

W

n

MW MN

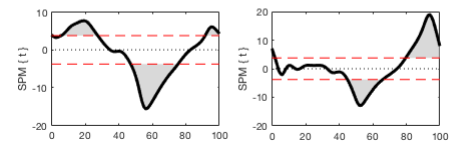

$m$ wh 

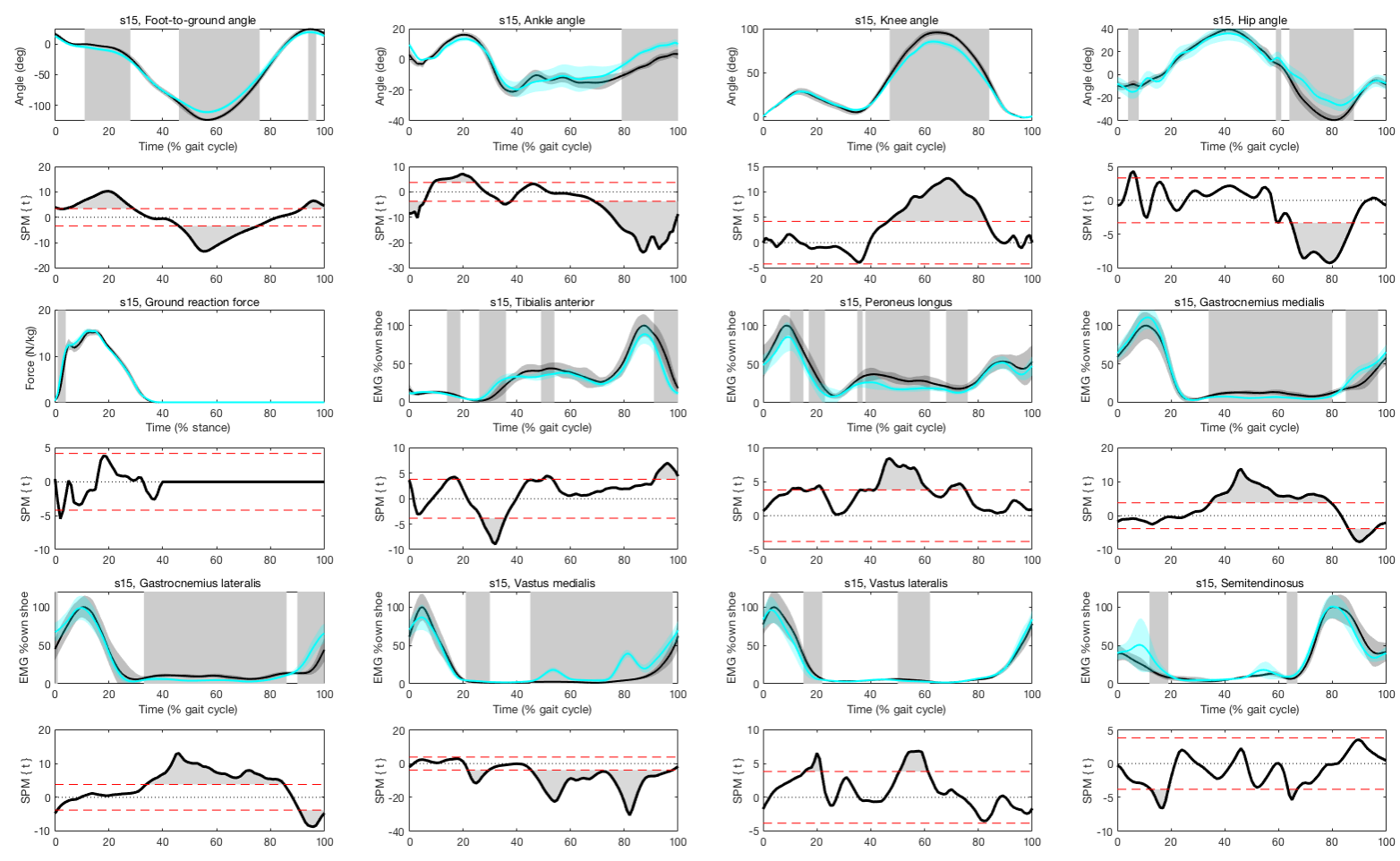

was 